4. - Les valeurs asymptotiques de quelques fonctions meromorphes dans le cercle-unite, C. R. Acad. Sci. Paris vol. 237 (1953) pp. 16-18.

5. N. Lusin and J. Privaloff, Sur l'unicite et la multiplicite des fonctions analytiques, Ann. Ecole Norm. (13) vol. 42 (1925) pp. 143-191.

6. R. Nevanlinna, Eindeutige analytische Funktionen, Berlin, Springer, 1936.

7. F. Riesz, Über die Randwerte einer analytischen Funktion, Math. Zeit. vol. 18 (1923).

UNIVERSity OF Michigan

\title{
MULTIPLICATIVE GROUPS OF ANALYTIC FUNCTIONS
}

\section{WALTER RUDIN}

Let $D$ be a proper subdomain of the Riemann sphere, and let $M(D)$ be the multiplicative group of all regular single-valued analytic functions on $D$ which have no zeros in $D$. It is known [1] that the algebraic structure of the ring $R(D)$ of all regular single-valued analytic functions on $D$ determines (and is determined by) the conformal type of $D$. In this paper we ask the question: what information about $D$ does the algebraic structure of $M(D)$ give, and, conversely, which properties of $D$ determine the algebraic structure of $M(D)$ ? The answer is, briefly, that $M\left(D_{1}\right)$ and $M\left(D_{2}\right)$ are isomorphic if and only if $D_{1}$ and $D_{2}$ have the same connectivity.

Here the connectivity of $D$ is $k$ if the complement of $D$ has $k$ components, and is $\infty$ if the complement of $D$ has infinitely many (countable or power of the continuum) components. The structure of $M(D)$ is described in more detail in the theorem below.

If we associate with each $f \in M(D)$ the function $g=f /|f|$ we obtain a subgroup (isomorphic to $M(D)$ ) of the multiplicative group $C(D)$ of all continuous functions from $D$ into the unit circumference. Such functions have been studied in great detail by Eilenberg [2]. It is worth noting that our theorem is valid if we replace $M(D)$ by $C(D)$, and that the proof is essentially the same; but it seems more interesting to stay within the smaller group.

Before stating the theorem, it is convenient to define two subgroups of $M(D)$.

(1) Fix a point $z_{0} \in D$ and let $G(D)$ be the set of all $f \in M(D)$ such that $f\left(z_{0}\right)=1$. Then $M(D)$ is the direct product of $G(D)$ and the multiplicative group of the nonzero complex numbers, and $G(D)$

Presented to the Society, April 24, 1954; received by the editors March 23, 1954. 
contains no elements of finite order. Without loss of generality we shall assume that $z_{0}=0$.

(2) Let $\Gamma(D)$ be the set of all $f \in G(D)$ for which the equation $g^{n}=f$ has a solution $g \in G(D)$, for every nonzero integer $n$. It is clear that, given $f$ and $n$, there is only one such $g$, and that $g \in \Gamma(D)$.

THEOREM. (i) The groups $\Gamma\left(D_{1}\right)$ and $\Gamma\left(D_{2}\right)$ are isomorphic for any two proper subdomains of the Riemann sphere.

(ii) $G(D)$ is (isomorphic to) the direct product of $\Gamma(D)$ and $G(D) / \Gamma(D)$.

(iii) If the complement of $D$ has $k+1$ components, then $G(D) / \Gamma(D)$ is the direct product of $k$ infinite cyclic groups.

(iv) If the complement of $D$ has infinitely many components, then $G(D) / \Gamma(D)$ is isomorphic to the additive group of all integer-valued functions on a countable space.

The proof will be broken up into several steps.

Step 1 . The structure of $\Gamma(D)$. Since we consider single-valued functions only, and since every $f \in \Gamma(D)$ has $n$th roots of all orders, we see that $f \in \Gamma(D)$ if and only if the total change in the argument of $f(z)$, as $z$ travels over any closed path in $D$, is zero. Hence $f \in \Gamma(D)$ if and only if $f$ has a single-valued logarithm in $D$, i.e., if $f$ is an exponential. Writing $f(z)=e^{o(z)}$, where $g$ is normalized by $g(0)=0$, the one-to-one correspondence $f \leftrightarrow g$ is an isomorphism between $\Gamma(D)$ and the additive group $A(D)$ of all single-valued regular analytic functions $g$ on $D$ such that $g(0)=0$. Part (i) of the theorem will follow if we can show that $A\left(D_{1}\right)$ and $A\left(D_{2}\right)$ are isomorphic.

We are going to show a little more. Let $L(D)$ be the vector space (over the complex field) whose members are the members of $A(D)$; we shall see that $L\left(D_{1}\right)$ and $L\left(D_{2}\right)$ are isomorphic.

Let $\operatorname{dim} L(D)$ stand for the cardinality of a Hamel basis of $L(D)$. It is enough to show that $\operatorname{dim} L(D)$ does not depend on $D$. Let $U$ be an open circular disc (the unit disc, without loss of generality) such that $U \subset D$, let $Z$ be the finite plane, and assume (again without loss of generality) that $D \subset Z$. Then

so that

$$
L(U) \supset L(D) \supset L(Z)
$$

$$
\operatorname{dim} L(U) \geqq \operatorname{dim} L(D) \geqq \operatorname{dim} L(Z) .
$$

To prove that the equality signs actually hold, we exhibit an isomorphism between $L(U)$ and a subspace of $L(Z)$ : to $f \in L(U)$, $f(z)=\sum_{1}^{\infty} a_{n} z^{n}$, associate the function $g \in L(Z)$ given by $g(z)$ $=\sum_{1}^{\infty} a_{n}(z / n)^{n}$. 
Having proved part (i) of the theorem, we shall from now on write $\Gamma$ in place of $\Gamma(D)$.

Step 2. Domains of finite connectivity. Suppose $D$ is bounded, and let $C_{1}, \cdots, C_{k}$ be the bounded components of the complement of $D$. Choose points $a_{i}(i=1, \cdots, k)$ in $C_{i}$. For every $f \in G(D)$ there is a unique set of integers $n_{1}, \cdots, n_{k}$ and a unique function $g \in \Gamma$ such that

$$
f(z)=g(z) \prod_{i=1}^{k}\left(1-\frac{z}{a_{i}}\right)^{n_{i}} .
$$

The integer $n_{j}$ is obtained by considering the change in arg $f(z)$ as $z$ travels around a simple closed curve which contains $C_{j}$ (and no other $\left.C_{i}\right)$ in its interior. The functions $\left(1-z / a_{i}\right)$ are the generators of the factor group $G(D) / \Gamma$.

The representation $\left({ }^{*}\right)$ proves the theorem completely, for domains of finite connectivity.

Step 3. The structure of $G(D) / \Gamma$ for domains of infinite connectivity. We shall assume that the complement $C$ of $D$ is bounded, and will construct a countable set of simple polygons $P_{t}$, with interiors $Q_{t}$, as follows. $Q_{1}$ contains $C . P_{11}$ and $P_{12}$ are in $Q_{1}$, are exterior to each other, $C \cap Q_{11} \neq 0, C \cap Q_{12} \neq 0$, and

$$
C=\left(C \cap Q_{11}\right) \cup\left(C \cap Q_{12}\right) .
$$

We continue in this manner: if, for some $t, Q_{t}$ contains only one component of $C$, we call $P_{t}$ a final polygon. If $P_{t}$ is not final, we construct $P_{10 t+1}$ and $P_{10 t+2}$ in $Q_{t}$, exterior to each other, such that $C \cap Q_{10 t+1} \neq 0, C \cap Q_{10 t+2} \neq 0$, and

$$
C \cap Q_{t}=\left(C \cap Q_{10 t+1}\right) \cup\left(C \cap Q_{10 t+2}\right) .
$$

We may construct these polygons so that they satisfy one further condition: for every $\delta>0$ there exists an integer $t_{0}$ such that $Q_{t}$ contains no two components of $C$ whose distance exceeds $\delta$ whenever $t>t_{0}$. (For the details of such constructions, we refer to $[3, \mathrm{pp} .46-$ 56].)

We let $T$ be the set of all $t$ such that $P_{t}$ is a nonfinal polygon of the set so constructed. $T$ is a countable set of integers (whose decimal representations consist of ones and twos), and is the space mentioned in part (iv) of the theorem.

Let $T^{\prime}$ be the set of all integers $t$ for which $P_{t}$ exists. For $t \in T^{\prime}$ and $f \in G(D)$, put

$$
2 \pi w_{f}(t)=\Delta_{\imath} \arg f(z),
$$


where $\Delta_{t}$ indicates the increment as $z$ travels around $P_{t}$ in the positive direction. Then $w_{f}(1)=0$, and for $t \in T$ the argument principle implies that

$$
w_{f}(10 t+1)+w_{f}(10 t+2)=w_{f}(t) .
$$

For $f \in G(D)$, we now define an integer-valued function $s_{f}$ on $T$ by:

$$
s_{f}(t)=w_{f}(10 t+1)-w_{f}(t)=-w_{f}(10 t+2) .
$$

Then $s_{f o}(t)=s_{f}(t)+s_{g}(t)$, so that the mapping $f \rightarrow s_{f}$ is a homomorphism of $G(D)$ into the additive group $S$ of all integer-valued functions on $T$. Since $s_{f}(t)=s_{g}(t)$ for all $t \in T$ if and only if $w_{f}(t)=w_{g}(t)$ for all $t \in T^{\prime}$, and this last equality occurs if and only if $f^{-1} g \in \Gamma$, we see that $\Gamma$ is the kernel of the above homomorphism, so that $G(D) / \Gamma$ is isomorphic to a subgroup of $S$.

We next wish to show that $G(D) / \Gamma$ is isomorphic to $S$, i.e., the homomorphism of $G(D)$ into $S$ is onto.

Let $s \in S$. We shall construct a function $f \in G(D)$ such that $s_{f}=s$. For $t \in T$, choose $x_{t} \in C \cap Q_{10 t+1}, y_{t} \in C \cap Q_{10 t+2}$, such that $\left|x_{t}-y_{t}\right| \rightarrow 0$ as $t \rightarrow \infty$ (this is possible, by the last condition which we imposed on the polygons). Putting

$$
u_{t}(z)=\left(x_{t}-y_{t}\right) /\left(z-x_{t}\right) \quad(t \in T, z \in D)
$$

we have $1-u_{t}(z)=\left(z-y_{t}\right) /\left(z-x_{t}\right)$. Since $x_{t}-y_{t} \rightarrow 0$, there exists a sequence of positive integers $k_{t}$ (for instance, $k_{t}=t+|s(t)|$ ) such that

$$
\sum_{t \in T} s(t)\left\{u_{t}(z)\right\}^{k_{t}}
$$

converges absolutely for $z \in D$, and uniformly in every closed subset of $D$. Like in the standard proof of the Weierstrass factorization theorem for entire functions we now see that the product

$$
g(z)=\prod_{t \in T}\left\{\left[1-u_{t}(z)\right] \exp \left[u_{t}(z)+\cdots+\frac{\left[u_{t}(z)\right] k_{t}-1}{k_{t}-1}\right]\right\}^{*(t)}
$$

defines a regular analytic function with no zeros in $D$. Putting $f(z)=g(z) / g(0)$, we obtain the desired function: $s_{f}(t)=s(t)$ for all $t \in T$.

This completes the proof of part (iv) of the theorem.

Step 4. $G(D)$ has $\Gamma$ as a direct factor. In the case of finite connectivity this was trivial; in the general case, we proceed as follows:

By Zorn's lemma, $G(D)$ contains a subgroup $H$ which is maximal with respect to the two properties 
(A) $\Gamma \cap H=\{1\}$ (the set consisting of the identity element alone);

(B) If $h \in G(D)$ and $h^{n} \in H$ for some integer $n \neq 0$, then $h \in H$.

By (A), the group $\Gamma H$ generated by $\Gamma$ and $H$ is the direct product of $\Gamma$ and $H$. Assume that $G(D)$ contains an element $f$ which is not in $\Gamma H$. Let $H(f)$ be the set of all $w \in G(D)$ such that $w^{m}=f^{n} h$ for some $h \in H$ and some integers $m, n(m \neq 0)$. Then $H(f)$ is a subgroup of $G(D)$ which contains $H$ properly and which satisfies (B). Since $H$ is maximal, $H(f)$ cannot satisfy (A), so that there exists an element $g_{0} \neq 1$ in $\Gamma$, and an integer $m \neq 0$, such that $g_{0}^{m}=f^{n} h$. Since $g_{0} \neq 1$, $g_{0}^{m} \neq 1$, so that $n \neq 0$ (by (A)). Choose $g \in \Gamma$ such that $g^{n}=g_{0}^{m}$; this is possible since $\Gamma$ is closed with respect to the operation of taking roots. Then $\left(g^{-1} f\right)^{n} \in H$, (B) implies that $g^{-1} f \in H$, but this contradicts the assumption that $f \notin \Gamma H$.

Thus $G(D)=\Gamma H$. This establishes part (ii) and completes the proof of the theorem.

We conclude with the following remark. Since $G(D)=\Gamma H$ and $G(D) / \Gamma$ is isomorphic to $S$, we see that $H$ and $S$ are isomorphic. Moreover, this isomorphism is induced by the natural mapping $f \rightarrow s_{f}$ of $H$ onto $S$. In Step 3 we constructed, for each $s \in S$, a function $f \in G(D)$ such that $s_{f}=s$; but the functions obtained by means of this construction do not form a group, and it seems difficult to modify the construction (by proper choice of convergence factors) so as to make the abstract considerations of Step 4 unnecessary.

\section{REFERENCES}

1. Lipman Bers, On rings of analytic functions, Bull. Amer. Math. Soc. vol. 54 (1948) pp. 311-315.

2. Samuel Eilenberg, Transformations continues en circonférence et la topologie $d u$ plan, Fund. Math. vol. 26 (1936) pp. 61-112.

3. B. v. Kerékjárto, Vorlesungen ìber Topologie, Berlin, 1923.

UNIVERSITY OF ROCHESTER 